\title{
On Generalized Carleson Operators of Periodic Wavelet Packet Expansions
}

\author{
Shyam Lal and Manoj Kumar \\ Department of Mathematics, Faculty of Science, Banaras Hindu University, Varanasi 221005, India \\ Correspondence should be addressed to Shyam Lal; shyam_lal@rediffmail.com
}

Received 29 April 2013; Accepted 14 July 2013

Academic Editors: M. Mansour and E. Momoniat

Copyright (c) 2013 S. Lal and M. Kumar. This is an open access article distributed under the Creative Commons Attribution License, which permits unrestricted use, distribution, and reproduction in any medium, provided the original work is properly cited.

Three new theorems based on the generalized Carleson operators for the periodic Walsh-type wavelet packets have been established. An application of these theorems as convergence a.e. for the periodic Walsh-type wavelet packet expansion of block function with the help of summation by arithmetic means has been studied.

\section{Introduction}

Wavelet packet expansions have wide applications in engineering and technology. The Walsh-type wavelet packet expansions play an important role in signal processing, numerical analysis, and quantum mechanics. A family of nonstationary wavelet packets considered the smooth generalization of the Walsh functions having some of the same nice convergence properties for expansion of $L^{p}$-function, $1<p<\infty$, as the Walsh-Fourier series. Walsh-type wavelet packet expansion has been studied by the researchers Billard [1], Nielsen [2], Sjölin [3] and others. In 1966, at first, Carleson operator has been introduced by Lennart Carleson (Carleson [4]). Several important properties of this operator has been studied by researcher Nielsen [2]. In this paper, the pointwise convergence almost everywhere by arithmetic means or $(C, 1)$ summability method of the partial sum operator for Walsh-type wavelet packet expansion of functions from the block space, $\mathbb{B}_{q}, 1<q \leq \infty, p^{-1}+$ $q^{-1}=1$ has been studied. Generalized Carleson operators are introduced and some new properties of generalized Carleson operators are investigated. Specific convergence properties of Walsh-type wavelet packet expansions of block functions using $(C, 1)$ method and generalized Carleson operator have been obtained.

\section{Definitions and Preliminaries}

Walsh-Type Wavelet Packets. To every multiresolution analysis $\left\{V_{j}\right\}_{j \in \mathbb{Z}}$ for $L^{2}(\mathbb{R})$, an associated scaling function $\varphi$ and a wavelet $\psi$ are given with the properties that

$$
\begin{gathered}
V_{j}=\overline{\operatorname{span}}\left\{2^{j / 2} \varphi\left(2^{j} \cdot-k\right): k \in \mathbb{Z}\right\}, \quad j \in \mathbb{Z}, \\
\left\{\psi_{j, k} \equiv 2^{j / 2} \psi\left(2^{j} \cdot-k\right): j, k \in \mathbb{Z}\right\}
\end{gathered}
$$

is an orthonormal basis for $L^{2}(\mathbb{R})$.

We write

$$
W_{j}=\overline{\operatorname{span}}\left\{2^{j / 2} \psi\left(2^{j} \cdot-k\right): k \in \mathbb{Z}\right\}, \quad j \in \mathbb{Z} .
$$

Let $\mathbb{N}$ be the set of natural numbers. Let $\left(F_{0}^{(p)}, F_{1}^{(p)}\right), p \in$ $\mathbb{N}$, be a family of bounded operators on $l^{2}(\mathbb{Z})$ of the form

$$
\left(F_{\epsilon}^{(p)} a\right)_{k}=\sum_{n \in \mathbb{Z}} a_{n} h_{\epsilon}^{(p)}(n-2 k), \quad \epsilon=0,1
$$

with $h_{1}^{(p)}(n)=(-1)^{n} h_{0}^{(p)}(1-n)$ a real-valued sequence in $l^{1}(\mathbb{Z})$ such that

$$
\begin{gathered}
F_{0}^{(p) *} F_{0}^{(p)}+F_{1}^{(p) *} F_{1}^{(p)}=1, \\
F_{0}^{(p)} F_{1}^{(p) *}=0 .
\end{gathered}
$$


Define the family of functions $\left\{w_{n}\right\}_{n=0}^{\infty}$ recursively by letting $w_{0}=\varphi, w_{1}=\psi$ and then for $n \in \mathbb{N}$,

$$
\begin{gathered}
w_{2 n}(x)=\sqrt{2} \sum_{l \in \mathbb{Z}} h_{0}^{(p)}(l) w_{n}(2 x-l), \\
w_{2 n+1}(x)=\sqrt{2} \sum_{l \in \mathbb{Z}} h_{1}^{(p)}(l) w_{n}(2 x-l),
\end{gathered}
$$

where $2^{p} \leq n<2^{p+1}$.

The family $\left\{w_{n}\right\}_{n=0}^{\infty}$ is basic non stationary wavelet packets. $\left\{w_{n}(\cdot-k): n \geq 0, k \in \mathbb{Z}\right\}$ is an orthonormal basis for $L^{2}(\mathbb{R})$.

Moreover,

$$
\left\{w_{n}(\cdot-k): 2^{j} \leq n<2^{j+1}, k \in \mathbb{Z}\right\}
$$

is an orthonormal basis for $W_{j}=\overline{\operatorname{span}}\left\{2^{j / 2} \psi\left(2^{j} \cdot-k\right): k \in \mathbb{Z}\right\}$.

Each pair $\left(F_{0}^{(p)}, F_{1}^{(p)}\right)$ can be chosen as a pair of quadrature mirror filters associated with a multiresolution analysis, but this is not necessary.

The trigonometric polynomials given by

$$
\begin{aligned}
& m_{0}^{(p)}(\xi)=\frac{1}{\sqrt{2}} \sum_{k \in \mathbb{Z}} h_{0}^{(p)}(k) e^{-i k \xi}, \\
& m_{1}^{(p)}(\xi)=\frac{1}{\sqrt{2}} \sum_{k \in \mathbb{Z}} h_{1}^{(p)}(k) e^{-i k \xi}
\end{aligned}
$$

are called the symbols of the filters.

The Fourier transforms of (5) are given by

$$
\begin{gathered}
\widehat{w}_{2 n}(\xi)=m_{0}^{(p)}\left(\frac{\xi}{2}\right) \widehat{w}_{n}\left(\frac{\xi}{2}\right), \\
\widehat{w}_{2 n+1}(\xi)=m_{1}^{(p)}\left(\frac{\xi}{2}\right) \widehat{w}_{n}\left(\frac{\xi}{2}\right) .
\end{gathered}
$$

The Haar low-pass quadrature mirror filter $\left\{h_{0}(k)\right\}_{k}$ is given by $h_{0}(0)=h_{0}(1)=1 / \sqrt{2}, h_{0}(k)=0$ otherwise, and the associated high-pass filter $\left\{h_{1}(k)\right\}_{k}$ is given by

$$
h_{1}(k)=(-1)^{k} h_{0}(1-k) .
$$

Definition 1. Let $\left\{w_{n}\right\}_{n \geq 0, k \in \mathbb{Z}}$ be a family of non-stationary wavelet packets constructed by using a family $\left\{h_{0}^{(p)}(n)\right\}_{p=1}^{\infty}$ of finite filters for which there is a constant, $K \in \mathbb{Z}$ such that $h_{0}^{(p)}(n)$ is the Haar filter for every $p \geq K$. If $w_{1} \in C^{1}(\mathbb{R})$ is compactly supported then $\left\{w_{n}\right\}_{n \geq 0}$ is called a family of Walshtype wavelet packets.

Definition 2. Let $\left\{w_{n}\right\}_{n=0}^{\infty}$ be a family of Walsh-type basic wavelet packets. For $n \in \mathbb{N}_{0}$, define the corresponding periodic Walsh-type wavelet packets $\widetilde{w}_{n}$ by

$$
\widetilde{w}_{n}(x)=\sum_{k \in \mathbb{Z}} w_{n}(x-k) .
$$

From Fubini's theorem, it follows that $\left\{\widetilde{w}_{n}\right\}_{n=0}^{\infty}$ is an orthonormal basis for $L^{2}[0,1)$.

Block Spaces. A dyadic $q$-block is a function $\beta \in L^{q}[0,1)$ which is supported on some dyadic interval $I$ such that $\|\beta\|_{q} \leq|I|^{1 / q-1}$, where $\|\beta\|_{q}=\left[\int_{0}^{1}|\beta(t)|^{q} d t\right]^{1 / q}, 1<q<\infty$. Let $\mathbb{B}_{q}$ denote the space of measurable functions $f$ on $[0,1)$ which has an expansion

$$
f=\sum_{k=1}^{\infty} c_{k} \beta_{k}
$$

where each $\beta_{k}$ is a $q$-block and the coefficients $c_{k}, k \in \mathbb{Z}$ satisfy

$$
\left\|\left\{c_{k}\right\}\left|\|=\sum_{k: c_{k} \neq 0}\right| c_{k} \mid\left[1+\log \frac{\sum_{j=1}^{\infty}\left|c_{j}\right|}{\left|c_{k}\right|}\right]<\infty .\right.
$$

The quasi norm of $f \in \mathbb{B}_{q}$ is given as the infimum of $\||\cdot|\|$ over all possible decompositions of $f$ into blocks

$$
\|f\|_{\mathbb{B}_{q}}=\inf _{f=\sum c_{k} \beta_{k}}\left\|\left\{c_{k}\right\}\right\| .
$$

Let $f \in \mathbb{B}_{q}$; then

$$
\|f\|_{1} \leq \sum_{k=1}^{\infty}\left|c_{k}\right|\left\|\beta_{k}\right\|_{1} \leq \sum_{k=1}^{\infty}\left|c_{k}\right|<\infty
$$

using (12) and the fact that for each $k,\left\|\beta_{k}\right\|_{q} \leq|I|^{1 / q-1}$ which implies that $\left\|\beta_{k}\right\|_{1} \leq 1$; that is, $\mathbb{B}_{q} \subset L^{1}[0,1)$. Moreover, for

$$
f \in L^{q}[0,1), \quad 1<q<\infty, \quad \beta=\|f\|_{q}^{-1} f
$$

is a $q$-block supported on $I=[0,1)$ so $L^{q}[0,1) \subset \mathbb{B}_{q}$.

The classical example to show that for each $q>1$ there exists $f \in \mathbb{B}_{q}$ which belongs to none of the $L^{p}[0,1)$-space is the following.

Let

$$
\beta_{k}(x)= \begin{cases}2^{k}, & \frac{1}{2^{k}}<x \leq \frac{3}{2^{(k+1)}}, \\ 0, & \text { otherwise. }\end{cases}
$$

Then $f=\sum_{k=1}^{\infty} k^{-2} \beta_{k} \in \mathbb{B}_{q}$, but $\|f\|_{p}^{p}=$ $\sum_{k=1}^{\infty}(1 / 2) k^{-2 p} 2^{k(p-1)}=\infty$ for every $p>1$.

Summation of Series by Arithmetic Means. If a series $u_{0}+u_{1}+$ $u_{2}+\cdots$ is not convergent, that is, if $s_{n}=u_{0}+u_{1}+u_{2}+\cdots+u_{n}$ does not tend to a limit, it is some time possible to associate with the series a "sum" in a less direct way. The simplest such method is "summation by arithmetic means". Let

$$
\sigma_{n}=\frac{s_{0}+s_{1}+s_{2}+\cdots+s_{n}}{n+1}
$$

be the arithmetic mean of the partial sums of the given series.

If $s_{n} \rightarrow s$, then also $\sigma_{n} \rightarrow s$; for if $s_{n}=s+\delta_{n}$, then

$$
\sigma_{n}=s+\frac{\delta_{0}+\delta_{1}+\delta_{2}+\delta_{2}+\cdots+\delta_{n}}{n+1},
$$


and the last term tends to zero if $\delta_{n} \rightarrow 0$. Consider

$$
\begin{aligned}
\sigma_{n}= & \frac{s_{0}+s_{1}+s_{2}+\cdots+s_{n}}{n+1} \\
= & \left(u_{0}+\left(u_{0}+u_{1}\right)+\cdots+\left(u_{0}+u_{1}+\cdots+u_{k}\right)\right. \\
& \left.\quad+\cdots+\left(u_{0}+u_{1}+\cdots+u_{n}\right)\right) \times(n+1)^{-1} \\
& \left.\quad+\frac{k}{n+1}\right) u_{k} .
\end{aligned}
$$

If $\sigma_{n} \rightarrow s$ as $n \rightarrow \infty, \sum_{n=0}^{\infty} u_{n}$ is said to be summable to $s$ by Cesàro's means of order 1 . We write

$$
\sum_{n=0}^{\infty} u_{n}=s(C, 1) .
$$

But $\sigma_{n}$ may tend to a limit even though $s_{n}$ does not, for example, the series

$$
1-1+1-1+\cdots
$$

Here the partial sums $s_{n}$ are alternately 1 and 0 , and it is easily seen that $\sigma_{n} \rightarrow 1 / 2$.

2.1. Generalized Carleson Operators. Let $\left\{\widetilde{w}_{n}\right\}$ be a periodic Walsh-type wavelet packet basis. For any function $f \in$ $L^{1}[0,1)$, define

$$
\left(S_{N} f\right)(x)=\sum_{n=0}^{N}\left\langle f, \widetilde{w}_{n}\right\rangle \widetilde{w}_{n}(x) .
$$

The Carleson operator $\mathbb{G}$ is defined by

$$
\begin{aligned}
\mathbb{G} f(x) & =\sup _{N \geq 0}\left|\sum_{n=0}^{N}\left\langle f, \widetilde{w}_{n}\right\rangle \widetilde{w}_{n}(x)\right| \\
& =\sup _{N \geq 0}\left|\left(S_{N} f\right)(x)\right| .
\end{aligned}
$$

The generalized Carleson operator $\mathbb{G}_{c}$ is defined by

$$
\begin{aligned}
\mathbb{G}_{c} f(x) & =\sup _{N \geq 0}\left|\frac{\left(S_{0} f\right)(x)+\left(S_{1} f\right)(x)+\cdots+\left(S_{N} f\right)(x)}{N+1}\right| \\
& =\sup _{N \geq 0}\left|\frac{1}{N+1} \sum_{\nu=0}^{N} \sum_{n=0}^{v}\left\langle f, \widetilde{w}_{n}\right\rangle \widetilde{w}_{n}(x)\right| \\
& =\sup _{N \geq 0}\left|\sum_{n=0}^{N}\left(1-\frac{n}{N+1}\right)\left\langle f, \widetilde{w}_{n}\right\rangle \widetilde{w}_{n}(x)\right| .
\end{aligned}
$$

The weak Carleson operator $G$ is defined by

$$
\begin{aligned}
G f(x) & =\limsup _{N \geq 0}\left|\sum_{n=0}^{N}\left\langle f, \widetilde{w}_{n}\right\rangle \widetilde{w}_{n}(x)\right| . \\
& =\limsup _{N \geq 0}\left|\left(S_{N} f\right)(x)\right| .
\end{aligned}
$$

The generalized weak Carleson operator $G_{c}$ is define by

$$
\begin{aligned}
G_{c} f(x) & =\limsup _{N \geq 0}\left|\frac{\left(S_{0} f\right)(x)+\left(S_{1} f\right)(x)+\cdots+\left(S_{N} f\right)(x)}{N+1}\right| \\
& =\limsup _{N \geq 0}\left|\frac{1}{N+1} \sum_{\nu=0}^{N} \sum_{n=0}^{v}\left\langle f, \widetilde{w}_{n}\right\rangle \widetilde{w}_{n}(x)\right| \\
& =\underset{N \geq 0}{\limsup }\left|\sum_{n=0}^{N}\left(1-\frac{n}{N+1}\right)\left\langle f, \widetilde{w}_{n}\right\rangle \widetilde{w}_{n}(x)\right| .
\end{aligned}
$$

The dyadic Carleson operator $\mathbb{G}^{d}$ is defined by

$$
\begin{aligned}
\mathbb{G}^{d} f(x) & =\sup _{N \geq 0}\left|\sum_{n=0}^{2^{N}-1}\left\langle f, \widetilde{w}_{n}\right\rangle \widetilde{w}_{n}(x)\right| \\
& =\sup _{N \geq 0}\left|\left(S_{2^{N}} f\right)(x)\right| .
\end{aligned}
$$

The generalized dyadic Carleson operator $\mathbb{G}_{c}^{d}$ is define by

$$
\begin{aligned}
\mathbb{G}_{c}^{d} f(x) & =\sup _{N \geq 0}\left|\frac{\left(S_{0} f\right)(x)+\left(S_{1} f\right)(x)+\cdots+\left(S_{2^{N}-1} f\right)(x)}{2^{N}}\right| \\
& =\sup _{N \geq 0}\left|\frac{1}{2^{N}} \sum_{v=0}^{2^{N}-1} \sum_{n=0}^{v}\left\langle f, \widetilde{w}_{n}\right\rangle \widetilde{w}_{n}(x)\right| \\
& =\sup _{N \geq 0}\left|\sum_{n=0}^{2^{N}-1}\left(1-\frac{n}{2^{N}}\right)\left\langle f, \widetilde{w}_{n}\right\rangle \widetilde{w}_{n}(x)\right| .
\end{aligned}
$$

It is easy to prove that $\mathbb{G}_{c}, G_{c}$ and $\mathbb{G}_{c}^{d}$ are sublinear operators.

Walsh Functions and Their Properties. The Walsh system $\left\{W_{n}\right\}_{n=0}^{\infty}$ is defined recursively on $[0,1)$ by letting

$$
\begin{gathered}
W_{0}(x)= \begin{cases}1, & 0 \leq x<1 ; \\
0, & \text { otherwise, }\end{cases} \\
W_{2 n}(x)=W_{n}(2 x)+W_{n}(2 x-1), \\
W_{2 n+1}(x)=W_{n}(2 x)-W_{n}(2 x-1) .
\end{gathered}
$$

Observe that the Walsh system is the family of wavelet packets obtained by considering $\varphi=W_{0}$,

$$
\psi(x)= \begin{cases}1, & 0 \leq x<\frac{1}{2} \\ -1, & \frac{1}{2} \leq x<1 \\ 0, & \text { otherwise }\end{cases}
$$

and using the Haar filters in the definition of the nonstationary wavelet packets.

The Walsh system is closed under pointwise multiplication. Define the binary operator $\oplus: \mathbb{N}_{0} \times \mathbb{N}_{0} \rightarrow \mathbb{N}_{0}$ by

$$
m \oplus n=\sum_{i=0}^{\infty}\left|m_{i}-n_{i}\right| 2^{i},
$$


where $m=\sum_{i=0}^{\infty} m_{i} 2^{i}$ and $n=\sum_{i=0}^{\infty} n_{i} 2^{i}$. Then

$$
W_{m}(x) W_{n}(x)=W_{m \oplus n}(x),
$$

(see Schipp et al. [5]).

We can carry over the operator $\oplus$ to the interval $[0,1]$ by identifying those $x \in[0,1]$ with a unique expansion $x=\sum_{j=0}^{\infty} x_{j} 2^{-j-1}$ (almost all $x \in[0,1]$ has such a unique expansion) by their associated binary sequence $\left\{x_{i}\right\}$. For two such points $x, y \in[0,1]$, define

$$
x \oplus y=\sum_{j=0}^{\infty}\left|x_{j}-y_{j}\right| 2^{-j-1} .
$$

The operation $\oplus$ is defined for almost all $x, y \in[0,1]$. With this definition, we have

$$
W_{n}(x \oplus y)=W_{n}(x) W_{n}(y)
$$

for every pair $x, y$ for which $x \oplus y$ is defined, (Golubov et al. [6], page 11).

\section{Main Results}

In this paper, three new theorems for the generalized Carleson operators on the periodic Walsh-type wavelet packets have been determined in the following form.

Theorem 3. Let $\left\{\widetilde{w}_{n}\right\}$ be a periodic Walsh-type wavelet packet basis. Then for every $q$-block $\beta, 1<q \leq \infty$,

$$
\left|\left\{\mathbb{G}_{c}^{d} \beta>\alpha\right\}\right| \leq \frac{C_{q}}{\alpha}, \quad \alpha>0,
$$

where $\mathbb{G}_{c}^{d}$ is the generalized dyadic Carleson operator defined by (28) and $C_{q}$ is a positive finite constant.

Theorem 4. Let $\left\{\widetilde{w}_{n}\right\}$ be a periodic Walsh-type wavelet packet basis. Then for every $q$-block $\beta, 1<q \leq \infty$,

$$
\left|\left\{G_{c} \beta>\alpha\right\}\right| \leq \frac{C_{q}}{\alpha}, \quad \alpha>0,
$$

where $G_{c}$ is the generalized weak Carleson operator defined by (26) and $C_{q}$ is a positive finite constant.

Theorem 5. If a function $f$ belongs to $\mathbb{B}_{q}$-class, $1<q \leq \infty$, then

$$
\left|\left\{G_{c} f>\alpha\right\}\right|=O\left(\|f\|_{\mathbb{B}_{q}}\right), \quad \text { for } \alpha>0,
$$

where $G_{c}$ is the generalized weak Carleson operator.

\section{Lemmas}

For the proof of our theorems, the following lemmas are required.
Lemma 6 (Nielsen [7]). Let $f_{1} \in L^{2}(\mathbb{R})$, and define $\left\{f_{n}\right\}_{n \geq 2}$ recursively by

$$
\begin{gathered}
f_{2 n}(x)=f_{n}(2 x)+f_{n}(2 x-1), \\
f_{2 n+1}(x)=f_{n}(2 x)-f_{n}(2 x-1) .
\end{gathered}
$$

Then

$$
f_{n}(x)=\sum_{s=0}^{2^{J}-1} W_{n-2^{J}}\left(s 2^{-J}\right) f_{1}\left(2^{J} x-s\right),
$$

where $n, J \in \mathbb{N}, 2^{J} \leq n<2^{J+1}$.

Lemma 7 (Zygmund [8], page 3). Consider

$$
\sum_{\nu=1}^{n} u_{\nu} v_{v}=\sum_{\nu=1}^{n-1}\left(v_{\nu}-v_{\nu+1}\right) U_{\nu}+U_{n} v_{n}
$$

where $U_{k}=u_{1}+u_{2}+\cdots+u_{k}$ for $k=1,2, \ldots, n$; it is also called Abel's transformation.

Lemma 8. Let $\left\{W_{n}\right\}_{n=0}^{\infty}$ be the Walsh system. Then

$$
\begin{aligned}
& \mid \sum_{n=2^{K}}^{m}\left(1-\frac{n}{m-2^{k}+1}\right) W_{n-2^{K}}\left(\left[2^{K} x\right] 2^{-K}\right) \\
& \quad \times W_{n-2^{K}}\left(\left[2^{K} y\right] 2^{-K}\right) \mid \\
& \leq \frac{C}{x \oplus y},
\end{aligned}
$$

where $C$ is a finite positive constant, $K \geq 1,2^{K} \leq n<2^{K+1 \text {, }}$ and for all pairs $x, y \in[0,1)$ for which $x \oplus y$ is defined.

Proof. The Dirichlet kernel, $D_{n}(x)=\sum_{k=0}^{n-1} W_{k}(x)$, for the Walsh system satisfies

$$
\left|D_{n}(x \oplus y)\right| \leq \frac{1}{x \oplus y}
$$

(see Golubov et al. [6], page 21).

Hence,

$$
\begin{gathered}
\mid \sum_{n=2^{K}}^{m}\left(1-\frac{n}{m-2^{k}+1}\right) W_{n-2^{K}}\left(\left[2^{K} x\right] 2^{-K}\right) \\
\quad \times W_{n-2^{K}}\left(\left[2^{K} y\right] 2^{-K}\right) \mid \\
=\mid \sum_{n=2^{K}}^{m}\left(1-\frac{n}{m-2^{k}+1}\right) W_{n-2^{K}} \\
\quad \times\left(\left[2^{K} x\right] 2^{-K} \oplus\left[2^{K} y\right] 2^{-K}\right) \mid
\end{gathered}
$$

$$
\begin{aligned}
=\mid \sum_{n=0}^{m-2^{K}}\left(1-\frac{n}{m-2^{k}+1}\right) W_{n} \\
\quad \times\left(\left[2^{K} x\right] 2^{-K} \oplus\left[2^{K} y\right] 2^{-K}\right) \mid
\end{aligned}
$$




$$
\begin{aligned}
=\mid & \sum_{n=0}^{m-2^{K}-1}\left\{\left(1-\frac{n}{m-2^{K}+1}\right)-\left(1-\frac{n+1}{m-2^{K}+1}\right)\right\} \\
& \times \sum_{r=0}^{n} W_{r}\left(\left[2^{K} x\right] 2^{-K} \oplus\left[2^{K} y\right] 2^{-K}\right) \\
& +\left(1-\frac{m-2^{K}}{m-2^{K}+1}\right) \\
& \times \sum_{n=0}^{m-2^{K}} W_{n}\left(\left[2^{K} x\right] 2^{-K} \oplus\left[2^{K} y\right] 2^{-K}\right) \mid,
\end{aligned}
$$

by Lemma 7 ,

$$
\begin{aligned}
& =\mid \sum_{n=0}^{m-2^{K}-1} \frac{1}{m-2^{K}+1} \\
& \times \sum_{r=0}^{n} W_{r}\left(\left[2^{K} x\right] 2^{-K} \oplus\left[2^{K} y\right] 2^{-K}\right)+\frac{1}{m-2^{K}+1} \\
& \times \sum_{n=0}^{m-2^{K}} W_{n}\left(\left[2^{K} x\right] 2^{-K} \oplus\left[2^{K} y\right] 2^{-K}\right) \\
& \leq \mid \sum_{n=0}^{m-2^{K}-1} W_{n}\left(\left[2^{K} x\right] 2^{-K} \oplus\left[2^{K} y\right] 2^{-K}\right)+\frac{1}{m-2^{K}+1} \\
& \times \sum_{n=0}^{m-2^{K}} W_{n}\left(\left[2^{K} x\right] 2^{-K} \oplus\left[2^{K} y\right] 2^{-K}\right) \\
& =\mid \sum_{n=2^{K}}^{m-1} W_{n-2^{K}}\left(\left[2^{K} x\right] 2^{-K} \oplus\left[2^{K} y\right] 2^{-K}\right)+\frac{1}{m-2^{K}+1} \\
& \times \sum_{n=2^{K}}^{m} W_{n-2^{K}}\left(\left[2^{K} x\right] 2^{-K} \oplus\left[2^{K} y\right] 2^{-K}\right) \\
& \leq\left|\sum_{n=2^{K}}^{m-1} W_{n-2^{K}}\left(\left[2^{K} x\right] 2^{-K} \oplus\left[2^{K} y\right] 2^{-K}\right)\right|+\frac{1}{m-2^{K}+1} \\
& \times\left|\sum_{n=2^{K}}^{m} W_{n-2^{K}}\left(\left[2^{K} x\right] 2^{-K} \oplus\left[2^{K} y\right] 2^{-K}\right)\right| \\
& =\mid W_{2^{K}}\left(\left[2^{K} x\right] 2^{-K} \oplus\left[2^{K} y\right] 2^{-K}\right) D_{m-2^{K}} \\
& \times\left(\left[2^{K} x\right] 2^{-K} \oplus\left[2^{K} y\right] 2^{-K}\right) \mid+\frac{1}{m-2^{K}+1} \\
& \times \mid W_{2^{K}}\left(\left[2^{K} x\right] 2^{-K} \oplus\left[2^{K} y\right] 2^{-K}\right) D_{m-2^{K}+1} \\
& \times\left(\left[2^{K} x\right] 2^{-K} \oplus\left[2^{K} y\right] 2^{-K}\right)
\end{aligned}
$$

$$
\begin{aligned}
= & \left|D_{m-2^{K}}(x \oplus y)\right| \\
& +\frac{1}{m-2^{K}+1}\left|D_{m-2^{K}+1}(x \oplus y)\right| \\
\leq & \frac{1}{(x \oplus y)}+\frac{1}{m-2^{k}+1} \frac{1}{(x \oplus y)}, \quad x \oplus y \neq 0 \\
= & \left(1+\frac{1}{m-2^{k}+1}\right) \frac{1}{(x \oplus y)} \\
\leq & \frac{C}{(x \oplus y)},
\end{aligned}
$$

where (32), (34), and the fact that $D_{\nu+1-2^{K}}$ is a constant on dyadic intervals of the form $\left[l 2^{-K},(l+1) 2^{-K}\right)$ are used. This completes the proof of Lemma 8 .

Lemma 9. If

$$
\begin{array}{r}
K_{J, m}^{(\sigma)}(x, y)=\sum_{n=2^{J}}^{m}\left(1-\frac{n}{m-2^{J}+1}\right) w_{n}(x) w_{n}(y), \\
\text { for } 2^{J} \leq m<2^{J+1},
\end{array}
$$

then

$$
\left|K_{J, m}^{(\sigma)}(x, y)\right| \leq \sum_{l=-2 N}^{2 N} \frac{C}{\left|x-y+2^{K-J} l\right|},
$$

where $C$ is an arbitrary constant.

Proof. The kernel can be expanded as

$$
\begin{gathered}
K_{J, m}^{(\sigma)}(x, y) \\
=\sum_{n=2^{J}}^{m}\left(1-\frac{n}{m-2^{J}+1}\right) w_{n}(x) w_{n}(y) \\
=\sum_{n=2^{J}}^{m}\left(1-\frac{n}{m-2^{J}+1}\right) \\
\times\left(\sum_{l=0}^{2^{J-K}-1} W_{n-2^{J-K}}\left(l 2^{-(J-K)}\right) w_{2^{K}}\left(2^{J-K} x-l\right)\right. \\
\quad \times \sum_{k=0}^{2^{J-K}-1} W_{n-2^{J-K}}\left(k 2^{-(J-K)}\right) \\
\left.\times w_{2^{K}}\left(2^{J-K} y-k\right)\right),
\end{gathered}
$$


by Lemma 6,

$$
\begin{aligned}
=\sum_{l=0}^{2^{J-K}-1} \sum_{k=0}^{2^{J-K}}\left\{\sum_{n=2^{J}}^{m}(1\right. & \left.-\frac{n}{m-2^{J}+1}\right) \\
& \times\left(W_{n-2^{J-K}}\left(l 2^{-(J-K)}\right)\right. \\
& \left.\times W_{n-2^{J-K}}\left(k 2^{-(J-K)}\right)\right) \\
& \times w_{2^{K}}\left(2^{J-K} x-l\right) \\
& \left.\times w_{2^{K}}\left(2^{J-K} y-k\right)\right\} .
\end{aligned}
$$

Therefore, using Lemma 8,

$$
\begin{gathered}
\left|K_{J, m}^{(\sigma)}(x, y)\right| \\
\leq \sum_{l=-N}^{N^{\prime}} \sum_{k=-N}^{N} \mid \sum_{n=2^{J}}^{m}\left(1-\frac{n}{m-2^{J}+1}\right) \\
\times W_{n-2^{J-K}}\left(\left[2^{J-K}\left(x+2^{K-J} l\right)\right] 2^{-(J-K)}\right) \\
\times W_{n-2^{J-K}}\left(\left[2^{J-K}\left(y+2^{K-J} k\right)\right] 2^{-(J-K)}\right) \mid \\
\leq \sum_{l=-N}^{N} \sum_{k=-N}^{N} \frac{C}{\left(x+2^{K-J} l\right) \oplus\left(y+2^{K-J} k\right)}
\end{gathered}
$$

where $\sum^{\prime}$ indicates that only the terms for which $x+2^{K-J} l \epsilon$ $[0,1)$ and $y+2^{K-J} k \in[0,1)$, respectively, should be included in the sum. This implies the estimate

$$
\left|K_{J, m}^{(\sigma)}(x, y)\right| \leq \sum_{l=-N}^{N} \sum_{k=-N}^{N} \frac{\widetilde{C}}{\left|x-y+2^{K-J}(l-k)\right|},
$$

since $a \oplus b \geq 2^{-\log _{2}[|a-b|]} \geq|a-b| / 2$. This completes the proof of Lemma 9.

\section{Proof of Theorem 3}

The dyadic arithmetic mean of partial sums for the expansion of a measurable (integrable) function $f$ in the periodic Walsh-type wavelet packets,

$$
\begin{aligned}
\left(\sigma_{2^{N}} f\right)(x) & =\frac{1}{2^{N}} \sum_{n=0}^{2^{N}-1}\left(S_{n} f\right)(x) \\
& =\frac{1}{2^{N}} \sum_{n=0}^{2^{N}-1}\left(\sum_{k=0}^{n}\left\langle f, \widetilde{w}_{k}\right\rangle \widetilde{w}_{k}(x)\right), \quad \text { by }(22), \\
& =\sum_{n=0}^{2^{N}-1}\left(1-\frac{n}{2^{N}}\right)\left\langle f, \widetilde{w}_{n}\right\rangle \widetilde{w}_{n}(x),
\end{aligned}
$$

holds everywhere with the arithmetic mean of the projection onto the (periodized) scaling space $\widetilde{V}_{N}$ associated with the underlying multiresolution analysis (Hess-Nielsen and Wickerhauser [9]). Therefore, it suffices to consider the arithmetic mean of the projection operators $P_{\widetilde{V}_{N}}$ on to the space $\widetilde{V}_{N}$.

Suppose that the $q$-block $\beta$ is associated with the dyadic interval $I \subset[0,1)$. If $1<\alpha|I|$, then $|I|^{1-q} / \alpha^{q} \leq 1 / \alpha$, and using the fact that the operator $f \rightarrow \sup _{N} \sum_{n=0}^{N}(1-n /(N+$ 1)) $P_{\widetilde{V}_{n}} f(x)$ (and thus $\left.f \rightarrow \mathbb{G}_{c}^{d} f(x)\right)$ is of strong type $(q, q)$. We have

$$
\left|\left\{\mathbb{G}_{c}^{d} f(x)>\alpha\right\}\right| \leq C_{q}\left(\frac{\|\beta\|_{q}}{\alpha}\right)^{q} \leq C_{q} \frac{|I|^{1-q}}{\alpha^{q}} \leq \frac{C_{q}}{\alpha} .
$$

Now suppose that $1 \geq \alpha|I|$ with $I=[a, b)$. Put $\tilde{I}=[(3 a-$ $b) / 2,(3 b-a) / 2] \cap[0,1)$, and define $\bar{I}=[0,1)-\widetilde{I}$. We have

$$
\begin{aligned}
\left|\left\{\mathbb{G}_{c}^{d} f(x)>\alpha\right\}\right| & \leq 2|I|+\left|\widetilde{I} \cap\left\{\mathbb{G}_{c}^{d} f(x)>\alpha\right\}\right| \\
& \leq \frac{2}{\alpha}+\left|\widetilde{I} \cap\left\{\mathbb{G}_{c}^{d} f(x)>\alpha\right\}\right| .
\end{aligned}
$$

Fix $x \in \bar{I}$, and let $K_{N}(x, y)$ denote the operator kernel associated with the projection operators $P_{\widetilde{V}_{N}}$. Then there exists a finite constant $C$ (independent of $N$ ) such that

$$
\left|K_{N}(x, y)\right| \leq \frac{C}{|x-y|}
$$

(see Terence [10]).

Using the estimate (52) on the kernel $K_{N}$, we obtain

$$
\left|\left(\sigma_{2^{N}} \beta\right)(x)\right|
$$

$$
\begin{aligned}
& =\left|\sum_{n=0}^{2^{N}-1}\left(1-\frac{n}{2^{N}}\right)\left\langle\beta, \widetilde{w}_{n}\right\rangle \widetilde{w}_{n}(x)\right| \\
& =\mid \sum_{n=0}^{2^{N}-2}\left\{\left(1-\frac{n}{2^{N}}\right)-\left(1-\frac{n+1}{2^{N}}\right)\right\} \\
& \quad \times \sum_{r=0}^{n}\left\langle\beta, \widetilde{w}_{r}\right\rangle \widetilde{w}_{r}(x) \\
& +\left(1-\frac{2^{N}-1}{2^{N}}\right) \sum_{n=0}^{2^{N}-1}\left\langle\beta, \widetilde{w}_{n}\right\rangle \widetilde{w}_{n}(x) \mid,
\end{aligned}
$$


by Lemma 7 ,

$$
\begin{aligned}
= & \mid \sum_{n=0}^{2^{N}-2} \frac{1}{2^{N}} \sum_{r=0}^{n}\left\langle\beta, \widetilde{w}_{r}\right\rangle \widetilde{w}_{r}(x) \\
& \quad+\frac{1}{2^{N}} \sum_{r=0}^{2^{N}-1}\left\langle\beta, \widetilde{w}_{n}\right\rangle \widetilde{w}_{n}(x) \mid \\
= & \left|\sum_{n=0}^{2^{N}-2} \frac{1}{2^{N}}\left(S_{n} \beta\right)(x)+\frac{1}{2^{N}}\left(S_{2^{N}} \beta\right)(x)\right| \\
\leq & \sum_{n=0}^{2^{N}-2} \frac{1}{2^{N}}\left|\left(S_{n} \beta\right)(x)\right|+\frac{1}{2^{N}}\left|\left(S_{2^{N}} \beta\right)(x)\right| \\
= & \left(\frac{2^{N}-2}{|x-a|}+\frac{C}{|x-b|}\right)\|\beta\|_{1} \cdot \\
\leq & \sum_{n=0}^{2^{N}} \frac{1}{2^{N}}\left|\int_{I} K_{n}(x, y) \beta(y) d y\right| \\
& +\frac{1}{2^{N}}\left|\sum_{n=0}^{2^{N}} \frac{1}{2^{N}}\left(\frac{C}{|x-a|}+\frac{C}{|x-b|}\right)\|\beta\|_{1}(x, y) \beta(y) d y\right|
\end{aligned}
$$

Since $\|\beta\|_{1} \leq 1$ and $x \in \bar{I}$ implies that $|x-a|,|x-b| \geq|I| / 2$, therefore,

$$
\begin{aligned}
\left|\left(\sigma_{2^{N}} \beta\right)(x)\right| & \leq\left\{\frac{2 C}{|I|}+\frac{2 C}{|I|}\right\} \\
& =\frac{4 C}{|I|} \leq \frac{\widetilde{C}}{\alpha} .
\end{aligned}
$$

Finally we obtain

$$
\left|\left\{x \in \bar{I}: \sup _{N}\left|\left(\sigma_{2^{N}} \beta\right)(x)\right|>\alpha\right\}\right| \leq \frac{\widetilde{C}}{\alpha},
$$

where $\widetilde{C}$ is independent of $I$ and $\beta$ and hence Theorem 3 follows.

\section{Proof of Theorem 4}

Fix $\alpha>0$ and a $q$-block $\beta$ supported on the dyadic interval $I \subset[0,1)$; two cases are considered.

Case I. If $1<\alpha|I|$, then $|I|^{1-q} / \alpha^{q} \leq 1 / \alpha$. Therefore, using Theorem 5.1. [7], page 275, we have

$$
\begin{aligned}
\left|\left\{G_{c} \beta>\alpha\right\}\right| & \leq C_{q}\left(\frac{\|\beta\|_{q}}{\alpha}\right)^{q} \\
& \leq C_{q} \frac{\left(|I|^{1 / q-1}\right)^{q}}{\alpha^{q}} \\
& =C_{q} \frac{|I|^{1-q}}{\alpha^{q}} \\
& \leq \frac{C_{q}}{\alpha}
\end{aligned}
$$

Case II. Let $1 \geq \alpha|I|$ with $I=[a, b)$. Let

$$
\widetilde{I}=\left(\cup_{j=-1}^{1}\left(j+\left[\frac{3 a-b}{2}, \frac{3 b-a}{2}\right)\right)\right) \cap[0,1),
$$

and define $\bar{I}=[0,1) \backslash \widetilde{I}$. Then

$$
\begin{aligned}
\left|\left\{G_{c} \beta>\alpha\right\}\right| & \leq|\widetilde{I}|+\left|\bar{I} \cap\left\{G_{c} \beta>\alpha\right\}\right| \\
& \leq 3|I|+\left|\bar{I} \cap\left\{G_{c} \beta>\alpha\right\}\right| \\
& \leq \frac{6}{\alpha}+\left|\bar{I} \cap\left\{G_{c} \beta>\alpha\right\}\right| .
\end{aligned}
$$

Notice that

$$
\begin{aligned}
\left|\bar{I} \cap\left\{G_{c} \beta>\alpha\right\}\right| \leq & \left|\widetilde{I} \cap\left\{G_{c}^{d} \beta>\frac{\alpha}{2}\right\}\right| \\
& +\left|\bar{I} \cap\left\{\underset{J}{\lim \sup } M_{J} \beta>\frac{\alpha}{2}\right\}\right|,
\end{aligned}
$$

with

$$
M_{J} \beta(x)=\max _{2^{J} \leq m<2^{J+1}-1} M_{J}^{m} \beta(x),
$$

$$
M_{J}^{m} \beta(x)=\left|\sum_{n=2^{J}}^{m}\left(1-\frac{n}{m-2^{J}+1}\right)\left\langle\beta, \widetilde{w}_{n}\right\rangle \widetilde{w}_{n}(x)\right| .
$$


For $x \in[0,1)$, we have

$$
\begin{aligned}
& \limsup M_{J}^{m} \beta(x) \\
& =\underset{J, m}{\lim \sup }\left|\sum_{n=2^{J}}^{m}\left(1-\frac{n}{m-2^{J}+1}\right)\left\langle\beta, \widetilde{w}_{n}\right\rangle \widetilde{w}_{n}(x)\right| \\
& =\underset{J, m}{\lim \sup } \mid \sum_{l_{1}=-N}^{N} \sum_{l_{2}=-N}^{N} \sum_{n=2^{J}}^{m}\left(1-\frac{n}{m-2^{J}+1}\right) \\
& \times\left\langle\beta, w_{n}\left(\cdot-l_{1}\right)\right\rangle w\left(x-l_{2}\right) \mid \\
& \leq\left.\sum_{l_{1}=-N}^{N} \sum_{l_{2}=-N}^{N} \limsup \right|_{J, m} \sum_{n=2^{J}}^{m}\left(1-\frac{n}{m-2^{J}+1}\right) \\
& \times\left\langle\beta, w_{n}\left(\cdot-l_{1}\right)\right\rangle w\left(x-l_{2}\right) \mid
\end{aligned}
$$

Hence, it suffices to estimate $\left|E_{\alpha}^{l_{1}, l_{2}}\right|$ with

$$
\begin{aligned}
& E_{\alpha}^{l_{1}, l_{2}} \\
& =\left\{x \in \bar{I}: \limsup _{J, m} \mid \sum_{n=2^{J}}^{m}\left(1-\frac{n}{m-2^{J}+1}\right)\right. \\
& \left.\quad \times\left\langle\beta, w_{n}\left(\cdot-l_{1}\right)\right\rangle w\left(x-l_{2}\right) \mid>\alpha\right\} .
\end{aligned}
$$

Fix $x \in \mathbb{R} \backslash I$; then

$$
\begin{aligned}
& \left|\int_{-\infty}^{\infty} K_{J, m}^{\sigma}\left(x-l_{1}, y-l_{2}\right) \beta(y) d y\right| \\
& \quad \leq \widetilde{C} \sum_{l=-2 N}^{2 N} \int_{-\infty}^{\infty} \frac{\beta(y) d y}{\left|x-y+l_{2}-l_{1}+2^{K-J} l\right|}
\end{aligned}
$$

which implies that whenever $x \in E_{\alpha}^{l_{1}, l_{2}}$, there is an increasing sequence $J_{k} \rightarrow \infty$ for which

$$
\begin{aligned}
& \left(\frac{1}{\mid x-a+l_{2}-l_{1}+2^{K-J_{k} l \mid}}\right) \\
& \left.+\frac{1}{\left|x-b+l_{2}-l_{1}+2^{K-J_{k} l}\right|}\right)>C \alpha,
\end{aligned}
$$

for some fixed $C>0$ and for $k=1,2, \ldots$ Since $J_{k} \rightarrow \infty$, therefore

$$
\left(\frac{1}{\left|x-a+l_{2}-l_{1}\right|}+\frac{1}{\left|x-b+l_{2}-l_{1}\right|}\right)>C \alpha .
$$

Using that $\bar{I}=[0,1) \backslash \widetilde{I}$ and the same technique as in the proof of Lemma 9, we complete the proof to conclude that $\left|E_{\alpha}^{l_{1}, l_{2}}\right| \leq 1 / \alpha$ and consequently

$$
\left|\bar{I} \cap\left\{\limsup _{J} M_{J} \beta>\frac{\alpha}{2}\right\}\right| \leq \frac{\widetilde{C}}{\alpha},
$$

which completes the proof of Theorem 4 .

\section{Proof of Theorem 5}

Let $f=\sum_{k=1}^{\infty} c_{k} \beta_{k}$ be a function of $\mathbb{B}_{q}$. Then

$$
\sigma_{N} f=\sum_{n=0}^{N}\left(1-\frac{n}{N+1}\right)\left\langle f, \widetilde{w}_{n}\right\rangle \widetilde{w}_{n}
$$

$$
\begin{aligned}
& =\sum_{n=0}^{N}\left(1-\frac{n}{N+1}\right)\left\langle\sum_{k=1}^{\infty} c_{k} \beta_{k}, \widetilde{w}_{n}\right\rangle \widetilde{w}_{n} \\
& =\sum_{n=0}^{N}\left(1-\frac{n}{N+1}\right) \sum_{k=1}^{\infty} c_{k}\left\langle\beta_{k}, \widetilde{w}_{n}\right\rangle \widetilde{w}_{n} \\
& =\sum_{k=1}^{\infty} c_{k}\left(\sum_{n=0}^{N}\left(1-\frac{n}{N+1}\right)\left\langle\beta_{k}, \widetilde{w}_{n}\right\rangle \widetilde{w}_{n}\right) \\
& =\sum_{k=1}^{\infty} c_{k}\left(\sigma_{N} \beta_{k}\right),
\end{aligned}
$$

due to the $L^{1}$ convergence of the average sum defining $f$. Since

$$
\begin{gathered}
\sum_{n=0}^{N}\left(1-\frac{n}{N+1}\right)\left\langle f, \widetilde{w}_{n}\right\rangle \widetilde{w}_{n} \\
=\sum_{k=1}^{\infty} c_{k} \sum_{n=0}^{N}\left(1-\frac{n}{N+1}\right)\left\langle\beta_{k}, \widetilde{w}_{n}\right\rangle \widetilde{w}_{n}, \\
\underset{N}{\limsup }\left|\sum_{n=0}^{N}\left(1-\frac{n}{N+1}\right)\left\langle f, \widetilde{w}_{n}\right\rangle \widetilde{w}_{n}\right| \\
\leq \sum_{k=1}^{\infty}\left|c_{k}\right| \limsup \left|\sum_{n=0}^{N}\left(1-\frac{n}{N+1}\right)\left\langle\beta_{k}, \widetilde{w}_{n}\right\rangle \widetilde{w}_{n}\right| \\
G_{c} f \leq \sum_{k=1}^{\infty}\left|c_{k}\right| G_{c} \beta_{k}
\end{gathered}
$$


therefore

$$
\begin{aligned}
\left|\left\{G_{c} f>\alpha\right\}\right| & \leq\left|\left\{\sum_{k=1}^{\infty}\left|c_{k}\right| G_{c} \beta_{k}>\alpha\right\}\right| \\
& \leq \frac{C_{q}}{\alpha} \sum_{k=1}^{\infty}\left|c_{k}\right|, \quad \text { by Theorem } 4, \\
& \leq \frac{C_{q}}{\alpha}\|f\|_{\mathbb{B}_{q}}, \quad \text { by }(12), \\
& =O\left(\|f\|_{\mathbb{B}_{q}}\right) .
\end{aligned}
$$

This completes the proof of Theorem 5 .

\section{Applications}

Following corollary can be deduced from our theorems.

Corollary 10. Let $\left\{\widetilde{w}_{n}\right\}$ be a periodic Walsh-type wavelet packet basis. Then the Fourier expansion of any function $f \in$ $\mathbb{B}_{q}, 1<q<\infty$, in $\left\{\widetilde{w}_{n}\right\}$ is summable by arithmetic means pointwise a.e.

Proof. Let us write $\left(S_{N} f\right)(x)=\sum_{n=0}^{N}\left\langle f, \widetilde{w}_{n}\right\rangle \widetilde{w}_{n}(x)$ and

$$
\begin{aligned}
\left(\sigma_{N} f\right)(x) & =\frac{1}{N+1} \sum_{n=0}^{N}\left(S_{n} f\right)(x) \\
& =\sum_{n=0}^{N}\left(1-\frac{n}{N+1}\right)\left\langle f, \widetilde{w}_{n}\right\rangle \widetilde{w}_{n}(x) .
\end{aligned}
$$

With $f=\sum_{k=1}^{\infty} c_{k} \beta_{k} \in \mathbb{B}_{q}$, let $g_{K}=\sum_{k=1}^{K} c_{k} \beta_{k}$, and observe that $\left\|f-g_{K}\right\|_{\mathbb{B}_{q}} \rightarrow 0$. For each $x \in[0,1)$, write

$$
\begin{aligned}
f-\sigma_{N} f= & \left(f-g_{K}\right) \\
& +\left(g_{K}-\sigma_{N} g_{K}\right)+\left(\sigma_{N} g_{K}-\sigma_{N} f\right) .
\end{aligned}
$$

Thus

$$
\begin{aligned}
& \left|\left\{x: \limsup _{N \rightarrow \infty}\left|f(x)-\left(\sigma_{N} f\right)(x)\right|>\alpha\right\}\right| \\
& \leq\left|\left\{x: \limsup _{N \rightarrow \infty}\left|f(x)-g_{K}(x)\right|>\frac{\alpha}{3}\right\}\right| \\
& \quad+\left|\left\{x: \limsup _{N \rightarrow \infty}\left|g_{K}(x)-\left(\sigma_{N} g_{K}\right)(x)\right|>\frac{\alpha}{3}\right\}\right| \\
& \quad+\left|\left\{x: \limsup _{n \rightarrow \infty}\left|\left(\sigma_{N} g_{K}\right)(x)-\left(\sigma_{N} f\right)(x)\right|>\frac{\alpha}{3}\right\}\right|
\end{aligned}
$$

$$
\begin{aligned}
& \leq\left|\left\{x: \limsup _{N \rightarrow \infty}\left|f(x)-g_{K}(x)\right|>\frac{\alpha}{3}\right\}\right| \\
& +\mid\left\{x: \limsup _{N \rightarrow \infty} \mid g_{K}(x)\right. \\
& \quad-\sum_{n=0}^{N}\left(1-\frac{n}{N+1}\right) \\
& \left.\quad \times\left\langle g_{K}, \widetilde{w}_{n}\right\rangle \widetilde{w}_{n}(x) \mid>\frac{\alpha}{3}\right\} \mid \\
& +\mid\left\{x: \limsup _{N \rightarrow \infty} \mid \sum_{n=0}^{N}\left(1-\frac{n}{N+1}\right)\right.
\end{aligned}
$$$$
\left.\times\left\langle g_{K}-f, \widetilde{w}_{n}\right\rangle \widetilde{w}_{n}(x) \mid>\frac{\alpha}{3}\right\} \mid
$$$$
\leq\left|\left\{x: \limsup _{N \rightarrow \infty}\left|f(x)-g_{K}(x)\right|>\frac{\alpha}{3}\right\}\right|
$$$$
+\left|\left\{x: \limsup _{N \rightarrow \infty}\left|g_{K}(x)-\sum_{n=0}^{N}\left\langle g_{K}, \widetilde{w}_{n}\right\rangle \widetilde{w}_{n}(x)\right|>\frac{\alpha}{3}\right\}\right|
$$$$
+\left|\left\{x: \limsup _{N \rightarrow \infty}\left|\sum_{n=0}^{N}\left\langle g_{K}-f, \widetilde{w}_{n}\right\rangle \widetilde{w}_{n}(x)\right|>\frac{\alpha}{3}\right\}\right|
$$$$
\leq\left|\left\{x: \limsup _{N \rightarrow \infty}\left|f(x)-g_{K}(x)\right|>\frac{\alpha}{3}\right\}\right|
$$$$
+\left|\left\{x: \limsup _{N \rightarrow \infty}\left|g_{K}(x)-\left(S_{N} g_{K}\right)(x)\right|>\frac{\alpha}{3}\right\}\right|
$$$$
+\left|\left\{x: \limsup _{N \rightarrow \infty}\left|\left(S_{N} g_{K}\right)(x)-\left(S_{N} f\right)(x)\right|>\frac{\alpha}{3}\right\}\right|
$$$$
\leq \frac{3}{\alpha}\left\|f-g_{K}\right\|_{\mathbb{B}_{q}}+0+\frac{3}{\alpha} C_{q}\left\|f-g_{K}\right\|_{\mathbb{B}_{q}}, \quad \text { by Theorem } 5 \text {. }
$$

From this it follows that

$$
\left|\left\{x: \limsup _{n \rightarrow \infty}\left|f(x)-\left(\sigma_{N} f\right)(x)\right|>\alpha\right\}\right|=0 .
$$

This completes the proof of the corollary.

\section{Acknowledgments}

Shyam Lal, one of the authors, is thankful to DST-CIMS for encouragement to this work. Manoj Kumar is grateful to CSIR, India in the form of Junior Research Fellowship vide Reference no. 17-06/2012 (i)EU-V dated 28-09-2012 for this research work.

\section{References}

[1] P. Billard, "Sur la convergence presque partout des séries de Fourier-Walsh des fontions de l'espace $L^{2}(0,1)$," Studia Mathematica, vol. 28, pp. 363-388, 1967. 
[2] M. Nielsen, Size Properties of Wavelet Packets [Ph.D. thesis], Washington University, St. Louis, Mo, USA, 1999.

[3] P. Sjölin, "An inequality of paley and convergence a.e. of WalshFourier series," in Arkiv för Matematik, vol. 7, pp. 551-570, 1969.

[4] L. Carleson, "On convergence and growth of partial sums of Fourier series," Acta Mathematica, vol. 116, no. 1, pp. 135-157, 1966.

[5] F. Schipp, W. R. Wade, and P. Simon, Walsh Series, Introduction to Dyadic Harmonic Analysis, Adam Hilger Ltd, Bristol, UK, 1990.

[6] B. Golubov, A. Efimov, and V. Skvortson, Walsh Series and Transforms: Theory and Application, Kluwer Academic, Dordrecht, The Netherlands, 1991, Translated From the 1987 Russian Original by W.R. Wade.

[7] M. Nielsen, "Walsh-type wavelet packet expansions," Applied and Computational Harmonic Analysis, vol. 9, no. 3, pp. 265$285,2000$.

[8] A. Zygmund, Trigonometric Series Volume I, Cambridge University Press, 1959.

[9] N. Hess-Nielsen and M. V. Wickerhauser, "Wavelets and timefrequency analysis," Proceedings of the IEEE, vol. 84, no. 4, pp. 523-540, 1996.

[10] T. Terence, "On the almost everywhere convergence of wavelet summation methods," Applied and Computational Harmonic Analysis, vol. 3, no. 4, pp. 384-387, 1996. 


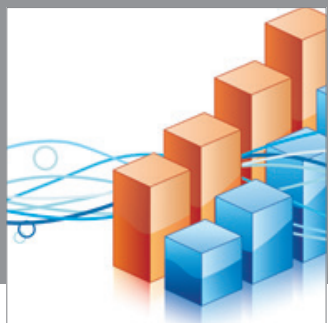

Advances in

Operations Research

mansans

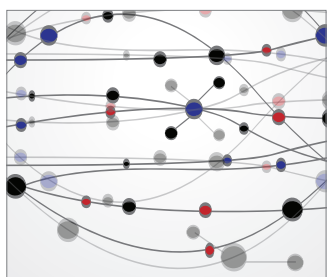

The Scientific World Journal
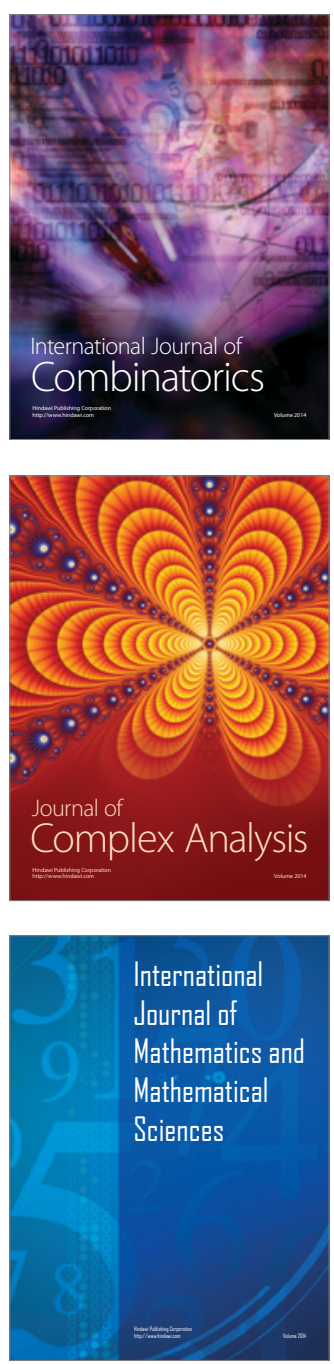
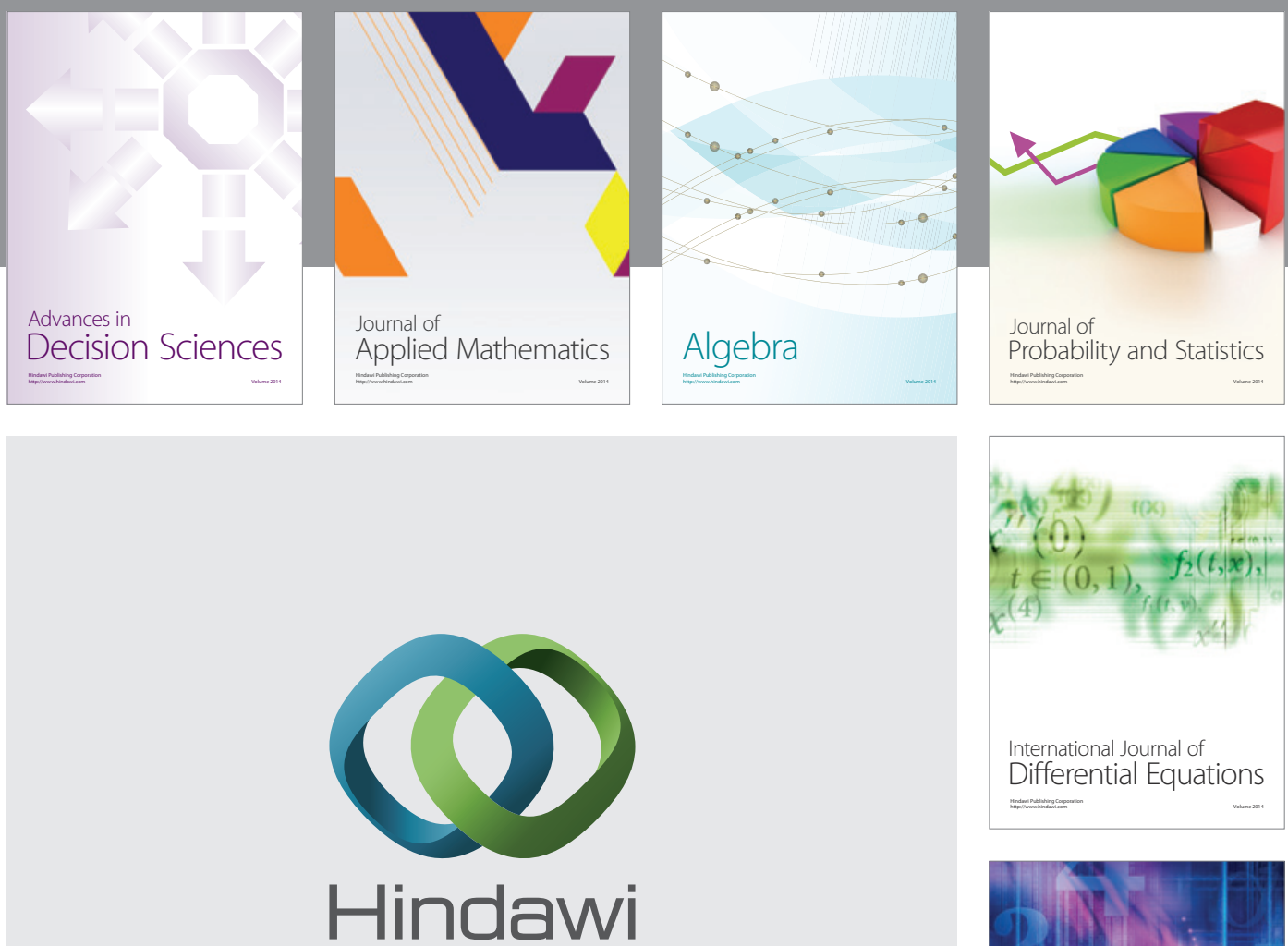

Submit your manuscripts at http://www.hindawi.com
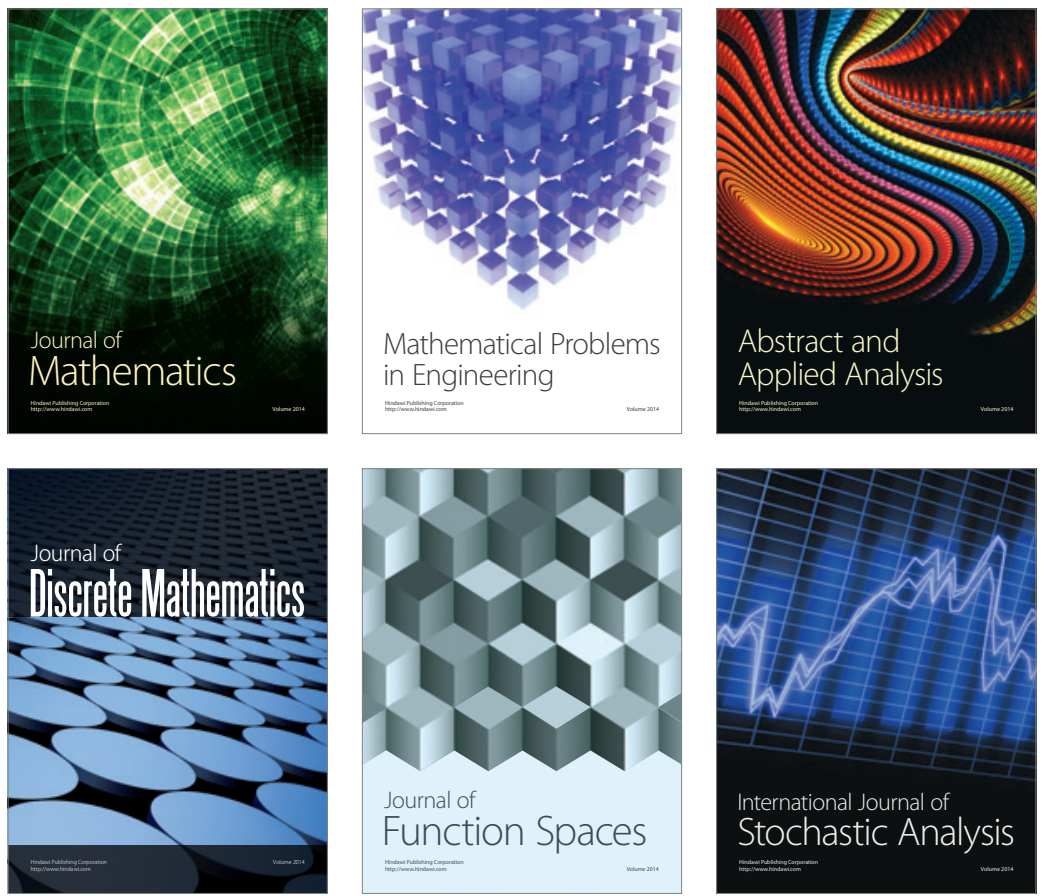

Journal of

Function Spaces

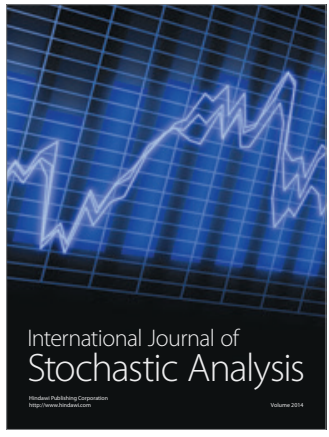

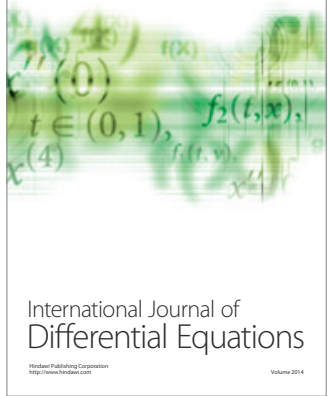
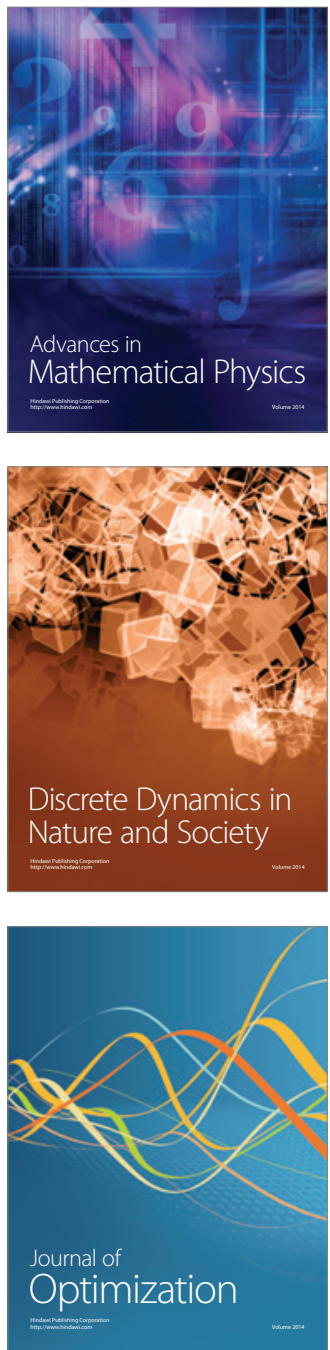